\title{
Evidence-based medicine clinical practice guidelines in the Spanish National Health System
}

\author{
Alberto Frutos Pérez-Surio, ${ }^{1,2}$ Ferran Sala-Piñol, ${ }^{3}$ Núria Sanmartí-Martinez ${ }^{4}$
}

\begin{abstract}
${ }^{1}$ Pharmacy Department, Hospital Clínico Universitario Lozano Blesa, Zaragoza, Aragón, Spain

2Department of Microbiology, Preventive Medicine and Public Health, Universidad de Zaragoza, Zaragoza, Aragón, Spain

${ }^{3}$ Pharmacy Department, Hospital Germans Trias i Pujol, Badalona, Cataluña, Spain ${ }^{4}$ Pharmacy Department, Hospital de Bellvitge, Hospitalet de Llobregat, Cataluña, Spain

\section{Correspondence to Professor Alberto Frutos Pérez-Surio, Pharmacy Department, Hospital Clínico Universitario Lozano Blesa, Avenida San Juan Bosco 15, Zaragoza, Aragón 50009, Spain; ajfrutos@salud.aragon. es}

EAHP STATEMENT 2: SELECTION, PROCUREMENT AND DISTRIBUTION EAHP STATEMENT 3: LOREM IPSUM DOLOR SIT AMET, CONSECTETUR ADIPISCING ELIT.

Received 29 October 2015 Revised 28 January 2016 Accepted 3 February 2016 Published Online First 26 February 2016

\begin{tabular}{l}
\hline To cite: Frutos Pérez- \\
Surio A, Sala-Piñol F, \\
Sanmartí-Martinez N. Eur J \\
Hosp Pharm 2016;23:308- \\
313. \\
\hline
\end{tabular}

\begin{abstract}
Spanish Hospital Pharmacies should become deeply implicated in clinical practice guidelines (CPGs) and other evidence-based products (OEBPs), either nationally or globally. The HealthGuide-CPG in the Spanish National Health System (NHS) is an organisation in which the 17 Spanish Autonomous Regions participate. It is endorsed by the Interterritorial Board in the NHS, as an instrument to improve the quality of care. The HealthGuide's mission is to encourage the supply of CPG and OEBP to support professionals and patients' decision making in the NHS and to promote the building of collaborative networks and the cooperation among entities dealing with CPGs and evidence-based medicine (EBM). The HealthGuide Technical Secretariat is located in the Aragon Health Sciences Institute. The aim of this paper is to present the HealthGuide to hospital pharmacists, not only to Spanishspeaking people, as it is applicable to any healthcare system. The resources proposed are methodological manuals for preparation, updating, implementation and patient involvement in the development of CPGs. The

\section{Key messages}

What is already known on this subject

- Clinical guidelines improve quality of healthcare and patients' safety.

- Public health administration leadership strengthen these projects.

- Evidence-based medicine (EBM) methodology supports scientific recommendations.

- EBM is a transversal interdisciplinary subject.

What this study adds

A comprehensive methodology to assist pharmacists interested in the appraisal, development, updating and implementation of clinical practice guidelines and a useful catalogue, with weblinks, of English-language guidelines that have merit as freestanding publications and permit international comparison with readers' own national guidelines.
\end{abstract} methods presented cover preparation, appraisal, updating, adapting and implementation of the CPG. Results Catalogue of CPGs for the Spanish NHS; programme development of CPGs in the NHS; OEBPs; and education and training for EBM and CPGs.

Discussion The Spanish hospital pharmacist should participate in the development of good CPGs, health technology assessment and OEBPs.

Conclusions The products and services developed in the HealthGuide framework may be a good way to acquire, improve and transfer methodological knowledge issues of EBM. The HealthGuide should take into account the knowledge of hospital pharmacists.

\section{INTRODUCTION}

At the end of 2012, two papers about evidencebased medicine (EBM) were published in the European Journal of Hospital Pharmacy (EJHP). ${ }^{12}$ In 2013, Wiffen et $a l^{3}$ published a Special Report in EJHP related to evidence-based pharmacy. Subsequent articles have been published in EJHP at approximately 2-month intervals until 2015. ${ }^{4-13}$ EBM is a core skill, sometimes not well known in many hospital pharmacy departments, and even misunderstood. English scientific and healthcare culture frameworks contribute to preparing initiatives based on EBM. Good examples are the National Institute for Health and Care Excellence (NICE), ${ }^{14}$ the Cochrane Collaboration, ${ }^{15}$ the Centre for Reviews and Dissemination (CRD) ${ }^{16}$ and the Centre for EBM (CEBM) at the University of Oxford. ${ }^{17}$ Our perception is that Spanish hospital pharmacies are not deeply implicated in many evidence-based products, either nationally or globally. There are many well prepared professionals who develop evidence-based practice (EBP) daily, and local or regional working groups contributing significantly to EBP.

In Spain, the HealthGuide Clinical Practice Guidelines (CPGs) in the Spanish National Health System (NHS), known in Spanish as GuíaSalud and available online at http://www.guiasalud.es, ${ }^{18}$ is an organisation of the Spanish NHS in which the 17 Autonomous Regions participate. It was created in 2002 and endorsed by the Interterritorial Board of the NHS as an instrument to improve the quality of care. The HealthGuide's mission is to encourage the supply of CPGs and other evidence-based products (OEBPs) to support professionals and patients' decision making in the NHS and to promote the creation of collaborative networks and cooperation among entities related to CPGs and EBM. The HealthGuide resembles NICE, the Scottish Intercollegiate Group Network (SIGN), and the National Guideline Clearinghouse (NGC) or Guidelines-International-Network (G-I-N) as a library of CPGs. Basically, it is a repository focused on Spanish documents. The HealthGuide Technical Secretariat is located in the Aragon Health Sciences Institute (Zaragoza, Spain).

The aim of this paper is to present the HealthGuide to hospital pharmacists, not only to Spanish-speaking people, as it is applicable to any healthcare system. 


\section{SCOPE AND OBJectives Definition}

Guideline Development Group (GDG)

Clinical Questions Formulation (PICO)

SCIENTIFIC LITERATURE SEARCH, EVALUATION AND SYNTHESIS

FORMULATION OF RECOMMENDATION

EXTERNAL REVISION

Glinical Practice Guideline Edition

Figure 1 Phases taken in developing process of clinical practice guideline.

\section{RESOURCES}

There are several methodological manuals proposed, mainly focused on CPGs and available on the HealthGuide web page (http://www.guiasalud.es), which are suitable as starting material for EBM research and development.

\section{Methodology manual for CPG preparation}

This methodology manual was designed with two objectives: to provide an agreed methodology for preparing CPGs developed with science-based evidence and to standardise the process for preparing CPGs based on this evidence.

The manual is organised according to the sequence of consecutive steps for CPG development (see figure 1). The manual's different sections are designed as independent units.

\section{Methodology manual for CPG updating}

This manual was designed by the HealthGuide as an instrument for updating CPGs developed as part of the CPG Programme in the Spanish NHS, to be used by any working group or institution which intends to update a CPG.

Updating should be understood as the process intended to ensure the continued validity and hence the quality of a CPG. Although there are few studies on this subject, it is considered that a CPG becomes obsolete after 3-5 years; therefore when this period has elapsed it should be updated.

It is assumed that there are two clearly differentiated stages:

- Monitoring to identify information indicating the need to update the CPG before the estimated maximum period (3-5 years after the end of systematic search).

- Updating process (literature search, critical appraisal, summary of the evidence, formulation of recommendations, editing of the CPG) starts when new evidence has been found as a result of monitoring, or when the maximum time set for updating the CPG has elapsed.

\section{Methodology manual for CPG implementation}

$\mathrm{CPG}$ implementation aims to ensure that the recommendations are followed; consequently any elements which may promote or obstruct this implementation must be identified.

This manual proposes a local adaptation process; therefore it is mandatory to know the characteristics of the context in which the CPG's recommendations are going to be applied.
The design of an implementation plan requires particular attention to be paid to institutional and social contexts in which a given CPG is going to be executed and also the barriers and facilitators which will hinder or promote the change in practice. Finally, it is also important to assess the intervention strategy that may be the most efficient for the successful implementation of the CPG.

\section{Methodology manual for patient involvement in CPG development}

The participation of professionals and patients in the process of elaboration of the CPG is essential to ensure its acceptance and application of the proposed recommendations. Depending on the level of participation and involvement of patients in the development of the CPG, important issues can be identified which are relevant to them and to help them understand the impact of health status over their lives. The CPG should focus on patients' main health outcomes, risks and benefits, and preferences about treatment and care. Additionally, professionals should explore patient information, their support needs, and understand their point of view and that of their families about their health condition.

The aim of this methodological manual is to provide tools, methods and strategies which facilitate the health (and nonhealth) professional's incorporation, and the participation of patients in the development of a CPG.

\section{METHODS}

The CPGs have the potential to facilitate deprescribing, reduce variability and improve clinical practice. The rigour and quality of CPG development has improved in recent years, especially since the publication of the Appraisal of Guidelines for Research and Evaluation (AGREE) instrument. In Spain, the launch of the Programme for Preparation of CPG in the NHS, coordinated by HealthGuide, led to a qualitative leap forward in CPG development. This programme, established by Ministerial agreement between the former NHS Quality Agency and the Spanish Network of Health Technology Assessment (HTA), involved a commitment to draw up a common methodology for CPG preparation, implementation and updating.

\section{Developing a CPG}

The development of a CPG consists of five phases: preparation; appraisal; updating; adaptation; and implementation of the CPG.

\section{Preparation of a CPG}

The final aim of this process is to develop a CPG using a systematic methodology with the phases shown in figure 1 .

A CPG may be developed for different reasons, including overprescription of drugs, variability in clinical practice due to the existence of areas of uncertainty; the existence of a significant health problem with impacts on morbidity and mortality; the development of new techniques, procedures or treatments; the possibility of achieving a change to improve healthcare outcomes as the process is capable of being improved through healthcare action. This is a priority area in the Spanish NHS.

\section{Appraisal of a CPG}

An activity intended to assess the quality of a CPG to ensure that its users can trust its recommendations when using it in their day-to-day clinical practice or, if a guideline development group (GDG) is in the process of adapting or updating a CPG, 
the ability to choose the best of several existing CPGs on the same subject. Different tools have been developed to assess the quality of CPGs for appraisal purposes. The AGREE Instrument is currently the most widely used internationally (http://www. agreetrust.org/).

\section{Updating a CPG}

A process intended to maintain the continued validity, and hence the quality, of a CPG. As previously mentioned, a CPG is estimated to be obsolete 3-5 years after its implementation, so when this period has elapsed it must be updated. Any CPG published or edited over 5 years ago which has not been updated will become an expired CPG.

\section{Adapting a CPG}

Preparing and updating CPGs are complex processes requiring effort, time and available resources. Adapting CPGs, therefore, is a good way to avoid duplicating effort, thereby improving efficiency in CPG development. There is an international initiative, the ADAPTE Group, which proposes adaptation as the use or modification of a CPG developed in a different cultural and organisational context, as an alternative to de novo guideline development. The group's proposal for adaptation is carried out according to a systematic process as shown in figure 2 .

\section{Implementation of a CPG}

This process aims to transfer CPG recommendations to daily practice. Implementation involves the use of effective communication strategies to bring about change. As previously stated, CPG implementation requires a planning process paying special attention to the context-both institutional and social; the barriers and facilitators which hinder or promote changes in practice; and the assessment of intervention strategies which, in the light of the above, may prove to be the most effective and efficient for implementing the CPG successfully. Assessment provides information on whether or not the strategies used to implement a CPG have been successful.

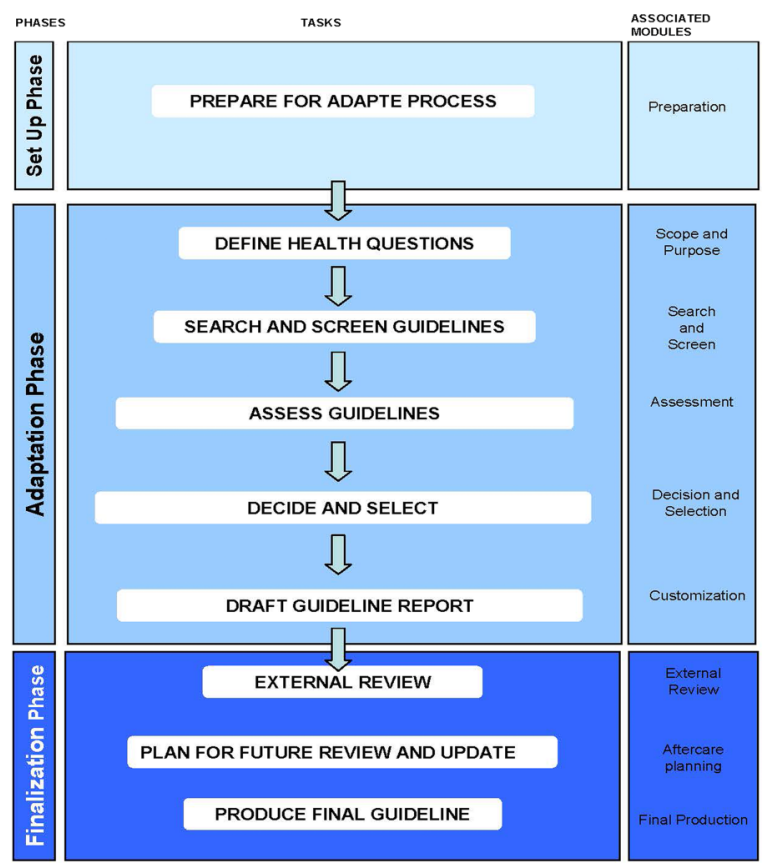

Figure 2 Practice guideline adaptation process.

\section{RESULTS}

The main products or services developed by HealthGuide are: Catalogue and Program Development of CPG in the NHS; OEBP; and Education and Training.

\section{Catalogue of CPG for the Spanish NHS}

Access to the full text of CPGs is included in the CPG Catalogue of the Spanish NHS. The guidelines are written in all of the official state languages in Spain by groups of professionals.

The appraisal of all CPGs in the HealthGuide Catalogue is based on six criteria for inclusion, as follows:

1. The document submitted must be a CPG. According to the Institutes of Medicine (IOM) CPG 2011 definition, ${ }^{19}$ the CPG is 'A set of recommendations based on a systematic review of the evidence and assessing the risks and benefits of different alternatives in order to optimise health care to patients'.

2. The guides must have been produced in Spain, in one of the official languages in the state; by public or private institutions, professional societies, expert panels and even professional groups; at the state, regional or local level. CPGs produced outside Spain are also accepted if they have been translated and backed by any of the previously mentioned institutions or groups.

3. The time interval between the closing date of the search for evidence and the timing of the inclusion of the CPG in the HealthGuide Catalogue must be less than 5 years.

4. In the CPG, author information, including name, qualifications (and specialty if applicable), institution and declaration of interest should appear.

5. The CPG has been developed, adapted or updated by following a proven methodology. The search methods used to obtain scientific evidence must be described, including search terms, sources consulted, the range of dates covered, inclusion and exclusion criteria, and the quality of the studies.

6. The recommendations of the CPG should be explicit and bibliographical sources that support them must be identified. Likewise, the recommendations should be formulated taking into account the benefits and potential risks of the different options assessed.

The Catalogue is continually updated based on requests for CPGs received by means of the Form for Inclusion of CPGs in the Catalogue.

The guidelines in the Catalogue are reviewed every 6 months to identify CPGs close to expiry, that is, CPGs which were published over 3 years previously without being updated. These data are used to establish a Work Plan to promote the updating of these CPGs among GDGs.

There are many examples ${ }^{20-23}$ of hospital pharmacists (or primary healthcare pharmacists) being involved in the development of good therapeutic guidelines. Other CPGs related to hospital pharmacy are GEICAM guide on clinical practice for the diagnosis and treatment of metastatic breast cancer (2015), CPG on comprehensive care for people with Alzheimer's disease and other dementias (2010), CPG on care for people with multiple sclerosis (2012), Clinic guide GETECCU of ulcerative colitis treatment made with the Grading of Recommendations Assessment, Development and Evaluation (GRADE) methodology (2012), or CPG for use of proton-pump inhibitor in secondary prevention of drug gastropathies (2012). GRADE is a working group that has developed a common, 
Box 1 Full versions of clinical practice guidelines (CPGs) translated into English within the CPG Programme

- CPG on the management of depression in adults (2014) http://www.guiasalud.es/contenidos/GPC/GPC 534 Depresion_Adulto_Avaliat_compl_en.pdf

- CPG for the management of invasive meningococcal disease (2013)http://portal.guiasalud.es/GPC/GPC_525_EMI_ICS_ compl_en.pdf

- CPG for diabetes mellitus type 1 (2012)http://www. guiasalud.es/GPC/GPC_513_Diabetes_1_0steba_compl_en. pdf

- CPG for the treatment of patients with chronic obstructive pulmonary disease (2012)http://www.guiasalud.es/GPC/GPC 512_EPOC_Lain_Entr_en.pdf

- CPG on sleep disorders in childhood and adolescence in primary care (2011)http://www.guiasalud.es/GPC/GPC_489_ Trastorno_sue\%C3\%B10_infadol_Lain_Entr_compl_en.pdf

- CPG on the comprehensive care of people with Alzheimer's disease and other dementias (2010)http://www.guiasalud.es/ GPC/GPC_484_Alzheimer_AIAQS_comp_eng.pdf

- GPC for urinary Tract infection in children (2011)http://www. guiasalud.es/GPC/GPC_483_ITU_poblacion_pediatrica_ICS_ ing.pdf

- CPG for the prevention and treatment of suicidal behaviour (2012)http://www.guiasalud.es/GPC/GPC_481_Conducta_ Suicida_Avaliat_compl_en.pdf

- CPG on attention deficit hyperactivity disorder in the child and adolescent population (2010)http://www.guiasalud.es/ GPC/GPC_477_TDAH_AIAQS_compl_en.pdf

- CPG on surgical patient safety (2010)http://www.guiasalud. es/GPC/GPC_478_Seguridad_Paciente_AIAQS_compl_en.pdf

- CPG on the diagnosis, treatment and prevention of tuberculosis (2010)http://www.guiasalud.es/GPC/GPC_473_ Tuberculosis_AIAQS_compl_en.pdf

- CPG on care for childbirth in healthy women (2010)http:// www.guiasalud.es/GPC/GPC_472_Parto_Normal_Osteba_ compl_en.pdf

- CPG on acute bronchiolitis (2010)http://www.guiasalud.es/ GPC/GPC_475_Bronchiolitis_AIAQS_compl_en.pdf

- GPC for the management of stroke patients in primary health care (2009)http://www.guiasalud.es/GPC/GPC_466_ Ictus_AP_Lain_Entr_compl_en.pdf

- CPG on prevention and treatment of childhood/adolescent obesity (2009)http://www.guiasalud.es/GPC/GPC_452_obes_ infantojuv_AATRM_compl_en.pdf

- GPC for the management of patients with insomnia in primary care (2009)http://www.guiasalud.es/GPC/GPC_465_ Insomnia_Lain_Entr_compl_en.pdf

- CPG on the management of patients with autistic spectrum disorder in primary health care (2009)http://portal.guiasalud. es/GPC/GPC_462_Autismo_Lain_Entr_compl_en.pdf

- CPG on major depression in childhood and adolescence (2009)http://portal.guiasalud.es/GPC/GPC_456_maj_dep_ child_adol_avaliat_compl_en.pdf

- CPG for psychosocial interventions in severe mental illness (2009)http://www.guiasalud.es/GPC/GPC_453_Severe_ Mental_Illness_ICS_compl_en.pdf sensible and transparent approach to grading quality of evidence and strength of recommendations.

\section{Programme development of CPGs in the NHS}

The objective of the CPG Programme in the Spanish NHS is 'To promote the preparation, adaptation and/or updating of CPGs based on the best scientific evidence available with a homogeneous and verified methodology'. It is also intended to distribute CPGs in the NHS.

This programme aims to develop the following:

- To coordinate the development of CPGs in the framework of the Spanish Network of HTAs.

- To advise methodological support and facilitate training for CPG development.

- To define methodology for CPG development (preparation, updating, implementation, prioritisation, etc).

- To develop CPGs based on scientific evidence.

- To facilitate access to CPGs in the NHS.

The CPG Programme has translated the full version of several CPGs into English. These are shown in box 1.

\section{Other evidence-based products}

OEBP development is carried out in the framework of the HealthGuide-CPG in the NHS. Its activities are focused on OEBPs other than CPGs which, given their characteristics, complement the guidelines for decision making in the health sector.

Its mission is to promote the use and production of information to guarantee quality care based on scientific criteria. The programme guarantees access to existing information and promotes the generation of new information, safeguarding quality at all times, so that it follows established standards and references.

The programme's objectives are:

- To promote the preparation, adaptation and updating of OEBPs, according to the needs of the NHS.

- To facilitate access to and use of a significant number of OEBPs to support the decisions made by the agents.

- To encourage the implementation and use of OEBPs.

- To assess the impact on health and continuous improvement of quality regarding applying OEBPs in the NHS.

- To promote training to prepare, introduce and assess the impact of OEBPs and to develop support tools for healthcare professionals.

- To promote research in the OEBP field.

OEBPs are instruments prepared using a systematic methodology which assists and guides professionals and healthcare service users in making the most appropriate decisions in a specific health or medical care situation.

Product definitions

1. Clinical questions: short documents prepared to help general practitioners to make decisions in specific situations, from a well defined clinical question and following a previously established system that includes the critical assessment of the available evidence. The 'PICO' approach is commonly used to formulate research questions; the acronym PICO stands for population (participants), intervention (or exposure for observational studies), comparison and outcomes.

2. Clinical pathways: this is an instrument aimed at structuring actions in the case of clinical situations with foreseeable evolution. It describes the steps which need to be taken, sets 
time sequences for each of them and defines the responsibilities of the different health professionals who intervene.

3. Protocols: documents aimed at facilitating clinical work, prepared by summarising information detailing the steps to be followed in the case of a specific healthcare problem. They are agreed among professionals, with 'agreement to comply' status and adapt to the context and the resources available.

Criteria which products must meet to be included in the HealthGuide

1. Clinical questions must have been prepared with the purpose of helping the clinician to make decisions in specific situations. Clinical pathways must have been prepared with the aim of sequencing and organising clinical work in predictable situations. Protocols must have been prepared with the aim of facilitating clinical work when faced with specific medical care problems.

2. Products must have been written in Spain, in any of the official state languages, by public or private institutions, scientific societies, expert panels and professional associations in the national, regional or local field. Those prepared abroad will also be accepted if they have been translated and/or endorsed by any of the previously mentioned institutions or groups.

3. The date of preparation, revision or update must be shown. No more than 5 years must have passed since the date of preparation, revision or update.

4. The authors' particulars-name, professional profile, institution, contact details-and conflict of interest statement should be clearly and explicitly shown.

5. The question must be formulated in PICO format and the search periods and sources used must be described. Description of the method of preparation must include the literature search and critical appraisal of the available

\section{Box 2 Desirable, scientific quality characteristics of} other evidence-based products

1. In its preparation, a strategy has been applied for searching studies in the relevant databases, following a pre-established method.

2. The evidence has been critically appraised, following a pre-established system (CASP, OSTEBA, SIGN, GRADE, etc). As an alternative to the two previous points, it has been prepared from an evidence-based guideline appraised with AGREE-II and classed as recommended or highly recommended.

3. It is multidisciplinary in nature, integrating the set of activities of all the professionals taking part.

4. An explicit and structured consensus method has been used in its preparation.

5. It is part of a quality improvement initiative or health plan/ programme.

6. Process and result indicators have been established to assess compliance and impact.

7. Diagrams, algorithms or other supporting instruments are included.

AGREE, Appraisal of Guidelines for Research and Evaluation; CASP, Critical Appraisal Skills Programme; GRADE, grading of recommendations assessment, development and evaluation; OSTEBA, Basque Office for Health Technology Assessment; SIGN, Scottish Intercollegiate Guidelines Network.
Table 1 Proposed modular activities that can be grouped under four training profiles to develop products with systematic and explicit methodology

Module 1. Introduction to EBM

Module 3. PICO: clinical question Module 2. Clinical practice guidelines

Module 5. Management of references Module 7. Experimental studies Module 4. Literature search Module 6. Epidemiological studies Module 8. Systematic reviews/reports of health technology assessment

Module 9. Diagnostic studies Module 11. Workshop AGREE Module 13. Qualitative research Module 15. Teach EBM

Module 10. Statistics Module 12. GRADE workshop Module 14. Decision making Module 16. Health technology assessment

Module 17. Health technology assessment. Economic evaluation Module 19. Bioethics, patient involvement and values and preferences

Module 18. Health technology assessment. Impact assessment Module 20. As lead groups or conduct meetings

AGREE, Appraisal of Guidelines for Research and Evaluation; EBM, evidence-based medicine; GRADE, Grading of Recommendations Assessment, Development and Evaluation; PICO, population/intervention/comparison/outcomes.

evidence or the steps taken must be specified if it has been prepared from a prior evidence-based guideline or document.

6. The response or proposed actions must be linked to the literature so that the studies which support them can be identified. This must include pictograms, matrices or diagrams identifying the main components, activities and times.

7. The field of application and the professionals for whom they are intended must be included.

Other desirable, scientific quality characteristics of OEBPs are shown in box 2 .

\section{Education and training}

Some services held and products developed include EBPs for information-related training activities, access to educational materials related to EBPs and proposals which serve as frameworks to develop training activities for EBPs. Training can refer to the following: scheduled courses and modules, training materials and training and education profiles. As suggested in the American Society of Health-System Pharmacists 'Guidelines on the pharmacist's role in providing drug information', the knowledge on pharmacotherapy and medication usage trends can be applied to support better guideline development. Pharmacists may also play a role in the teaching of EBM as they have medication expertise. Suggested reading might be the NICE guideline 'Medicines optimisation: the safe and effective use of medicines to enable the best possible outcomes'.

\section{Modules and profiles scheduled for EBPs}

The HealthGuide proposes a framework of training activities to develop products with systematic and explicit methodology. This education and training is organised in modular activities which enable training according to four profiles:

1. Basic training in CPGs and randomised controlled trials.

2. Advanced training for CPG development and other products for EBM.

3. Training for the development of systematic reviews and HTAs.

4. Training the trainers in how to teach EBM. 
The Spanish hospital pharmacist should participate in the development of good CPGs, HTAs and OEBPs. Table 1 shows proposed modular activities grouped under these training profiles.

\section{CONCLUSION}

The products and services developed in the HealthGuide framework include a comprehensive methodology to assist pharmacists interested in the appraisal, development, updating and implementation of CPGs and a useful catalogue, with weblinks, of English-language guidelines that have merit as freestanding publications and for international comparison with readers' own national guidelines. Most CPGs include pharmacotherapy or patient safety constructs, but few of them have a hospital pharmacist in their GDG. The HealthGuide should also take into account the knowledge of the hospital pharmacy.

Acknowledgements The authors would like to acknowledge the HealthGuide Technical Secretariat.

Competing interests AFP-S was a former member of the Aragon Health Sciences Institute and the HealthGuide, member of the GDG and coordinator of the CPG on the management of Parkinson's disease, 23 available at: http://www.guiasalud.es/ GPC/GPC_546_Parkinson_IACS_compl.pdf.

Provenance and peer review Not commissioned; externally peer reviewed.

\section{REFERENCES}

1 Wiffen P. Evidence-based medicine: tools not rules. Eur J Hosp Pharm 2012;19:498-501.

2 Moore RA, Derry S, Strassels SA. Knowing when evidence is trustworthy. Eur J Hosp Pharm 2012;19:507-9.

3 Wiffen $P$, Eriksson $T$, Lu H. Ensuring pharmacy practice is fit for purpose in evidence-based pharmacy 2nd edition. Eur J Hosp Pharm 2013;20:308-12.

4 Eriksson T, Lu H, Wiffen P. Chapter 8: generating knowledge. Eur J Hosp Pharm 2014;22:2-6.

5 Lu H, Eriksson T, Wiffen P. Chapter 9: evidence based pharmacy for developing countries. Eur J Hosp Pharm 2015;22:66-72.

6 Wiffen P, Eriksson T, Lu H. Chapter 3: asking and formulating the right questions and finding useful resources in evidence-based pharmacy 2 nd edition. Eur J Hosp Pharm 2014;21:2-6.
7 Wiffen P, Eriksson T, Lu H. Chapter 7: managing knowledge in evidence-based pharmacy. Eur J Hosp Pharm 2014;21:320-4.

8 Wiffen P, Eriksson T, Lu H. Chapter 5: appraising the evidence. Eur J Hosp Pharm 2014;21:134-8.

9 Eriksson T, Lu H, Wiffen P. Chapter 6: how to best practice evidence-based pharmacy with your available resources? Eur J Hosp Pharm 2014;21:194-201.

10 Wiffen P, Eriksson T, Lu H. Chapter 2: introduction to evidence-based practice in evidence-based pharmacy 2nd edition. Eur J Hosp Pharm 2013:20:324-7.

11 Wiffen P, Eriksson T, Lu H. Chapter 11: getting a GRIP on pharmacy services. Eur J Hosp Pharm 2015:22:252-4.

12 Wiffen P, Eriksson T, Lu H. Chapter 10: mentoring and teaching of evidence-based pharmacy. Eur J Hosp Pharm 2015:22:191-3.

13 Eriksson T, Wiffen P, Lu H. Chapter 12: evidence-based skills and practices for the future of pharmacy. Euro J Hosp Pharm 2015;22:314-17.

14 National Institute for Health and Care Excellence. http://www.nice.org.uk/ (accessed Jul 2015).

15 The Cochrane Collaboration. http://www.cochrane.org/ (accessed Jul 2015).

16 The University of York, Centre for Reviews and Dissemination. http://www.york.ac. uk/inst/crd/ (accessed Jul 2015).

17 University of Oxford, Centre for Evidence Based Medicine. http://www.cebm.net/ (accessed Jul 2015).

18 GuíaSalud-Biblioteca de Guías de Práctica Clínica del Sistema Nacional de Salud. http://www.guiasalud.es (accessed Jul 2015).

19 Committee on Standards for Developing Trustworthy Clinical Practice Guidelines, Board on Health Care Services and Institute of Medicine. Clinical practice guidelines we can trust. Washington DC: National Academies Press, 2011.

20 Fernández Urrusuno R, Serrano Martino C, Corral Baena S. Guía de Terapéutica Antimicrobiana del Área Aljarafe. Sevilla, 2012

21 Working Group of the Clinical Practice Guideline on the Management of Depression in Adults. Clinical Practice Guideline on the Management of Depression in Adults. Ministry of Health, Social Services and Equality. Galician Agency for Health Technology Assessment (avalia-t), 2014. SNS Clinical Practice Guidelines: Avalia-t 2013/06

22 Working Group of the Clinical Practice Guideline on Diabetes mellitus type 1. Clinical Practice Guidelines for Diabetes Mellitus type 1. Quality Plan for the National Health Service of the Ministry of Health and Social Policy. Agency for Health Technology Assessment from the Basque Country-Osteba, 2012. Clinical Practice Guidelines in the NHS: OSTEBA no. 2009/10.

23 Working Group of the Clinical Practice Guideline on the Management of Patients with Parkinson's Disease. Ministry of Health, Social Services and Equality. Aragonese Institute for Health Sciences, 2014. Clinical Practice Guidelines in the NHS. 